\title{
Violencia física en el embarazo: realidad en el extremo sur de Chile
}

\author{
Silvana Estefó A. MSc ${ }^{1 a}$, Sara Mendoza-Parra.MSc, PhD ${ }^{2 a}$, Katia Sáez C. MSc, PhD. ${ }^{3 b}$ \\ 1 Departamento de Enfermería, Facultad Ciencias de la Salud. Universidad de Magallannes, Punta Arenas. ${ }^{2}$ Departa- \\ mento de Enfermería, Facultad de Medicina. Universidad de Concepción, Concepción. ${ }^{3}$ Departamento de Estadística, \\ Facultad de Ciencias Físicas y Matemáticas, Universidad de Concepción, Concepción. Chile.
}

$\mathrm{a}_{\text {Enfermera. }}{ }^{\mathrm{b}}$ Ingeniero Matemático.

\section{RESUMEN}

Objetivo: Describir el perfil de las mujeres adscritas al Programa de Violencia, Punta Arenas, Chile, y que han presentado violencia física durante el embarazo. Método: Estudio de abordaje cuantitativo, con diseño descriptivo y retrospectivo. Se entrevistó a 59 mujeres, utilizando el Instrumento de la OMS sobre la violencia contra las mujeres Versión 9.1 para Chile modificado. Resultados: La prevalencia de violencia durante el embarazo fue $28,8 \%$. En su mayoría el agresor fue la pareja y el $52,9 \%$ declaró que los golpes o patadas fueron en el abdomen. El 100\% presento violencia psicológica, física leve y grave y $70,6 \%$ violencia sexual. El 58,8\% tenía entre 15 a 29 años al momento de vivenciar la violencia, el 35,5\% completó la enseñanza media, de religión católica, trabajan fuera del hogar, convivían, tenían tres o más hijos y con estrato socioeconómico medio y medio bajo. Las manifestaciones de violencia que se relacionaron con violencia física en el embarazo fueron la presencia de manifestaciones de violencia física leve: abofeteado o tirado cosas que pudieran herirla $(p=0,000)$ y violencia física grave: golpeado con su puño u otra cosa que pudiera herirla $(p=0,006)$, pateado, arrastrado o dado una golpiza $(p=0,004)$, estrangularla a propósito $(p=0,010)$, presencia de lesiones $(p=0,048)$. Los factores que se relacionaron con la presencia de violencia física en el embarazo fueron el nivel educacional de la pareja $(p=0,047)$, condición laboral de la mujer $(p=0,018)$ y antecedentes de peleas físicas en la pareja con otros hombres $(p=0,046)$. Conclusión: Los hallazgos obtenidos permiten identificar precozmente a las embarazadas con mayor riesgo de violencia, y permiten ofrecer una intervención oportuna, minimizando las graves consecuencias que tiene para la mujer y los hijos.

\section{PALABRAS CLAVE: Violencia contra la mujer, violencia física, factores de riesgo, embarazo}

\section{SUMMARY}

Aim: To describe the profile of the women assigned to the Violence Program who have submitted physical violence during pregnancy in Punta Arenas, Chile. Methods: Retrospective descriptive study with a quantitative approach. 59 women were interviewed using the WHO instrument on violence against women, modified Version 9.1 for Chile. Results: Prevalence of violence during pregnancy corresponded to $28.8 \%$. Most of the time, the aggressor was the intimate partner and $52.9 \%$ declared that the blows or kicks were in the abdomen. $100 \%$ presented severe and mild physical and psychological violence, and $70.6 \%$ presented sexual violence. $58.8 \%$ were between 15 and 29 years at the time of experiencing violence. $35,5 \%$ were catholic women who have finished high school, worked outside home, lived together with an intimate partner, had three or more children, and belonged to a middle and lower middle socioeconomic strata. Manifestations of violence which were related to physical violence during pregnancy were slapping or throwing things that could hurt them $(p=0.000)$. Severe physical violence included punching or being hurt with something that could hurt them $(p=0.006)$; kicking, dragging on the ground or being beaten $(p=0.004)$; strangling on purpose $(p=0.010)$, and appearance of injuries $(p=0.048)$. Factors related to manifestations 
of physical violence in pregnancy were: intimate partner's educational level (graduated high school or less than high school education $(p=0.047)$, employment status of women $(p=0.018)$ and the intimate partner history ofphysical fights with other men $(p=0,046)$. Conclusion: The findings obtained in this study give us the opportunity to visualize the phenomenon of physical violence during pregnancy, contributing to early identification of pregnant women at increased risk in order to provide timely intervention, minimizing the serious consequences for women and children.

\section{KEY WORDS: Violence against women, physical violence, risk factors, pregnancy}

\section{INTRODUCCIÓN}

La violencia de pareja representa un tipo de violencia al interior de la familia que ha estado presente en nuestra sociedad desde sus inicios. En la mayoría de los casos corresponde a violencia ejercida por el hombre hacia la mujer, por lo que en declaraciones internacionales (1), convenciones (2) y políticas a nivel nacional (3), ha sido abordada utilizando términos como violencia contra la mujer o violencia de género. En Chile, se define violencia de género (4), como aquella ejercida hacia la mujer por el simple hecho de serlo y que tiene como resultado posible o real un daño físico, sexual, psicológico y emocional.

La violencia contra las mujeres no cesa incluso durante el embarazo (5), la agresión es la continuación o intensificación de abusos anteriores, aunque en otros casos, la violencia se inicia en el momento en que las mujeres quedan embarazadas (6). En la literatura internacional, la prevalencia de violencia durante el embarazo varía entre $4 \%$ y $25 \%$ según la población, las definiciones de violencia, y los métodos utilizados para medirla (7). En Brasil la prevalencia oscila entre el 7,0-34\% $(8,9)$, Costa Rica 29,7\% (10), Estados Unidos 0,9-20,1\%, Canadá $5,7-8,5 \%$ y México 35\% (11). Estas realidades revelan, además que existen factores que se relacionan con este tipo de violencia, como antecedentes de violencia en la infancia de la mujer y pareja $(12,13,14)$, consumo de alcohol por parte de la pareja (12-14), situación conyugal (14) y nivel socioeconómico (15), entre otros.

Los estudios realizados en Chile, el primero en 1998 , en un consultorio de la ciudad de Valdivia (16) presentó una prevalencia de violencia durante el embarazo del $22,2 \%$. En 2001, un estudio en 422 familias en Temuco (17), reveló que el $8,5 \%$ de las mujeres habían presentado violencia física en el embarazo. El 2006 un estudio del SERNAM, en la región de los Lagos (18) informó que la prevalencia alcanzó el $16 \%$, y otro estudio el 2007 , en el Hospital Clínico Regional de Valdivia (19) esta cifra fue de $13,2 \%$. Del mismo modo, se encontró que la violencia durante el embarazo, estaba relacionada con el aborto espontáneo, el inicio tardío del control prenatal, la muerte prenatal, el parto y nacimientos prematuros, y el bajo peso al nacer (20).

Teniendo presente ésta realidad que no ha sido explorada en el extremo sur de Chile, el objetivo del estudio fue describir el perfil de las mujeres que han presentado violencia física en el embarazo, adscritas a los programas de violencia en la ciudad de Punta Arenas, determinando prevalencia, tipos de violencia y factores relacionados.

\section{SUJETOS Y MÉTODOS}

Tipo de estudio. Estudio de tipo cuantitativo, con un diseño descriptivo y retrospectivo.

Unidad de análisis. Estuvo conformada por cada una de las mujeres adscritas como víctimas al Programa de Violencia Intrafamiliar en los Centros de Salud Familiar y Centro de la mujer del SERNAM de la ciudad de Punta Arenas, ingresadas de enero a julio del año 2013.

Población. Estuvo conformada por 70 mujeres adscritas como víctimas al Programa de Violencia Intrafamiliar en los Centros de Salud Familiar (21) y 163 mujeres pertenecientes al Centro de la mujer del SERNAM de la ciudad de Punta Arenas (22) constituida por mujeres mayores de 15 años, que ingresaron a ambos programas entre los meses de enero y julio de 2013.

Plan de muestreo para las entrevistas. Se contactó personalmente al total de las mujeres ingresadas al programa de violencia, vía telefónica, se les explicó el motivo de la llamada y el propósito de la entrevista, si accedía a participar, se citaba a entrevista en el centro de la mujer o centro de salud familiar, se les dio también la opción de visitarla en su domicilio; 56 de ellas acudieron a entrevista a algunos de los centros, en horario y día de acuerdo a la disponibilidad de cada una de ellas y a 3 mujeres se les realizó la entrevista en el domicilio.

Muestra. Se logró entrevistar a 59 mujeres que alguna vez habían estado embarazadas, de las cuales 17 de ellas, presentaron violencia física durante el embarazo.

Recolección de los datos. Para la obtención de 
los datos, se utilizó el registro de ingreso 2013, al programa de violencia de los distintos centros. El instrumento de recolección de información fue el Cuestionario denominado Instrumento de la OMS sobre la violencia contra las mujeres. Versión 9.1 para Chile modificado. Esta nueva versión del instrumento, fue el resultado de la revisión de la versión 1.3 adaptada a Chile, que fue utilizada en 6 estudios en diversas regiones del país y la versión 10, que fue actualizada en el año 2005 y que ha sido utilizada solo en inglés en las ciudades de Maldivas, Nueva Zelanda y Serbia. Para efectos de este estudio el instrumento se tradujo al español y se adaptaron algunas preguntas a la realidad chilena, teniendo como referencia la versión 9 adaptada a Chile (23). Este instrumento consta en una primera parte de preguntas generales, dirigidas principalmente a evaluar el estrato socioeconómico, sector poblacional, la segunda parte está dividida en 12 secciones. Para efectos de este estudio se utilizaron algunas de las preguntas de las secciones.

Persona encuestada y su pareja: La violencia física durante el embarazo, se construyó a partir de las siguientes preguntas ¿Alguna vez cuando usted estuvo embarazada, fue agredida físicamente por alguna de sus parejas? ¿Esto ha pasado en un embarazo o en más de uno? ¿Ocurrió en el último embarazo? ¿Fue usted golpeada o pateada en el abdomen mientras estaba embarazada? ¿Durante el último embarazo en el cual fue golpeada o abofeteada, la persona que la golpeó, fue el padre del niño? Comparando con antes de que usted estuviera embarazada, ¿Los golpes o abofeteadas, disminuyeron, siguieron igual o aumentaron mientras estaba embarazada? ¿Alguna vez ha quedado con lesiones como resultado de la violenciadesu pareja? ¿Sus hijos estuvieron presentes durante los episodios de violencia?

Los actos violentos ejercidos en contra de la mujer se pueden presentar como distintos tipos de violencia, en términos globales las preguntas se orientaron a:

Violencia psicológica: El reconocimiento en las mujeres de haber vivido más de una vez situaciones en que la pareja actual o cualquier otra pareja la haya insultado o hecho sentir mal con ella misma, menospreciado o humillado frente a otras personas, hecho cosas a propósito para asustarla o intimidarla y amenazado con herirla a ella o a otra persona importante para ella.

Violencia física: Aquellas mujeres que mencionaron, al menos una vez, alguna situación en que su pareja actual o cualquier otra pareja la había abofeteado o tirado cosas que pudieran herirla, empujado, arrinconado o tirado el pelo, golpeado con su puño o con alguna otra cosa que pudiera herirla, pateado, arrastrado o dado una golpiza, intentado estrangularla, intentado quemarla o quemado, amenazado con usar o usado una pis- tola, cuchillo u otra arma en contra de ella.

Violencia sexual: Todas aquellas mujeres que mencionaron que al menos una vez su pareja actual o cualquier otra pareja la había forzado a tener relaciones sexuales cuando ella no lo deseaba, tuvo relaciones sexuales por miedo a lo que él pudiera hacer y forzado a realizar algún acto sexual que ella encontró humillante o degradante (24). Los tipos de violencia fueron indagados en cualquier momento de la relación de pareja.

Encuesta, comunidad y pareja actual o más reciente: Preguntas que se relacionaron con factores individuales de la mujer y de la pareja, como edad, nivel educacional, situación conyugal, consumo de alcohol, número de hijos.

Procesamiento de los datos: Se codificaron, depuraron y procesaron computacionalmente. Se construyó una base de datos en programa Excel. El análisis descriptivo y relacional, se realizó mediante el Programa Estadístico SPSS versión 21.0, con licencia de la Universidad de Magallanes.

Análisis de los datos. Se realizó un análisis descriptivo, representando las variables numéricas por sus medidas de tendencia central y de dispersión (promedio y desviación estándar) y las variables categóricas por su frecuencia y porcentaje, lo cual permitió identificar los tipos de violencia y caracterizar a la población en estudio. Para determinar la asociación entre la violencia física durante el embarazo y los factores sociodemográficos, antecedentes de violencia en la infancia, tipos y manifestaciones de violencia, se utilizó el test chi-cuadrado (test exacto de Fisher), con un nivel de significancia del 0,05.

Las consideraciones éticas se abordaron teniendo como base de referencia los siete requisitos éticos de Emmanuel y cols (25), las recomendaciones de la OMS (26) y los componentes éticos esenciales en la violencia, planteado por Arratia (27). Principalmente se resguardaron la privacidad, confidencialidad, seguridad, respeto y minimización de los riesgos. Las participantes firmaron un consentimiento informado, permitiéndole libremente decidir su participación. Este estudio conto la aprobación del Comité de Ética de la Facultad de Medicina de la Universidad de Concepción, autoridades de salud y del Servicio Nacional de la Mujer de Magallanes, Chile.

\section{RESULTADOS}

Prevalencia. De las 59 mujeres que han presentado violencia por parte de su pareja y que alguna vez estuvieron embarazadas, el $28,8 \%$ de ellas, presentó violencia física durante el embarazo.

Perfil sociodemográfico (Tabla I). El rango de edad que mayoritariamente se presentó fue 15 a 29 años, la mayoría completo la enseñanza media, pertenecían a la religión católica, trabajaban remuneradamente fuera del hogar y consumían 
alcohol menos de una vez al mes o nunca. Las diferencias en las mayorías porcentuales en ambos grupos, se presentaron en el número de hijos. El $70 \%$ de las mujeres que presentaron violencia física en el embarazo tenían 3 o más hijos, en cambio en las mujeres que no presentaron violencia física en el embarazo, el mayor porcentaje $(47,6 \%)$ tenían dos hijos. En relación a la situación conyugal, el $52,9 \%$ de las mujeres que presentaron violencia física en el embarazo, se encontraban conviviendo, en cambio el grupo que no presentaba violencia física en el embarazo, el 59,5\% estaban casadas. Características de la violencia física durante el

\section{Tabla I}

\section{PERFIL DE LAS MUJERES VIOLENTADAS POR SU PAREJA, SEGÚN PRESENCIA DE VIOLENCIA FíSICA DURANTE EL EMBARAZO, PUNTA ARENAS 2014 ( $n=59)$}

\begin{tabular}{|c|c|c|c|c|}
\hline \multirow[b]{2}{*}{ Variable } & \multicolumn{2}{|c|}{$\begin{array}{c}\text { Sin violencia física durante el } \\
\text { embarazo }\end{array}$} & \multicolumn{2}{|c|}{$\begin{array}{l}\text { Con violencia física durante } \\
\text { el embarazo }\end{array}$} \\
\hline & $\mathrm{n}$ & $\%$ & $\mathrm{n}$ & $\%$ \\
\hline \multicolumn{5}{|l|}{ Edad } \\
\hline 15 a 29 años & 27 & 64,3 & 10 & 58,8 \\
\hline 30 a 39 años & 7 & 16,7 & 5 & 29,4 \\
\hline 40 a 49 años & 7 & 16,7 & 2 & 11,8 \\
\hline 50 o más años & 1 & 2,4 & 0 & 0,0 \\
\hline \multicolumn{5}{|l|}{ Nivel educacional } \\
\hline Básica completa o menos & 10 & 23,8 & 5 & 29,5 \\
\hline Media incompleta & 5 & 11,9 & 3 & 17,6 \\
\hline Media completa & 21 & 50,0 & 6 & 35,3 \\
\hline Superior incompleta o completa & 6 & 14,3 & 3 & 17,6 \\
\hline \multicolumn{5}{|l|}{ Religión } \\
\hline Católica & 33 & 78,6 & 10 & 58,8 \\
\hline Otras & 5 & 11,9 & 4 & 23,5 \\
\hline No tiene & 4 & 9,5 & 3 & 17,6 \\
\hline \multicolumn{5}{|l|}{ Condición laboral } \\
\hline Trabajo remunerado fuera del hogar & 21 & 50,0 & 10 & 58,8 \\
\hline Trabajo remunerado en casa & 2 & 4,8 & 5 & 29,4 \\
\hline Trabajo remunerado esporádico & 4 & 9,5 & 0 & 0,0 \\
\hline Sin actividad & 15 & 35,7 & 2 & 11,8 \\
\hline \multicolumn{5}{|l|}{ Consumo de alcohol } \\
\hline Una o dos veces a la semana & 0 & 0,0 & 1 & 5,9 \\
\hline $1-3$ veces al mes & 13 & 31,0 & 4 & 23,5 \\
\hline Menos de una vez al mes & 16 & 38,0 & 6 & 35,3 \\
\hline Nunca & 13 & 31,0 & 6 & 35,3 \\
\hline \multicolumn{5}{|l|}{ Número de hijos } \\
\hline Un hijo & 4 & 9,5 & 2 & 11,8 \\
\hline Dos hijos & 20 & 47,6 & 3 & 17,6 \\
\hline Tres o más hijos & 18 & 42,9 & 12 & 70,6 \\
\hline \multicolumn{5}{|l|}{ Situación conyugal } \\
\hline Conviviente & 16 & 38,1 & 9 & 52,9 \\
\hline Casada & 25 & 59,5 & 8 & 47,1 \\
\hline Separada/pololo & 1 & 2,4 & 0 & 0,0 \\
\hline \multicolumn{5}{|l|}{ Estrato socio-económico } \\
\hline Muy Alto, Alto-medio & 10 & 23,8 & 2 & 11,8 \\
\hline Medio, Medio-bajo & 26 & 61,9 & 14 & 82,4 \\
\hline Bajo, Muy-bajo & 6 & 14,3 & 1 & 5,9 \\
\hline
\end{tabular}


embarazo (Tabla II). Se destaca, que del total de mujeres agredidas físicamente durante el embarazo, el $76,4 \%$ ocurrió en un embarazo y en el $52,9 \%$ esta agresión ocurrió en el último embarazo. Relacionado con lo anterior, esta agresión fue perpetrada casi en su mayoría por el padre del hijo que esperaba y el $88,2 \%$ de ellas vivía con esta pareja cuando sucedió la agresión. En un poco más de la mitad de las mujeres embarazadas, los golpes o patadas fueron en el abdomen y en el $82,3 \%$ la pareja la había golpeado anteriormente. La agresión física en la mujer durante el embarazo, permaneció igual en la mitad de ellas y en un $35,7 \%$ empeoró y en su mayoría los hijos estaban presentes. La totalidad de las mujeres que habían presentado violencia física durante el embarazo, el $100 \%$ había experimentado violencia física leve y grave. La manifestación de violencia física leve, "la han abofeteado o tirado cosas que pudieron herirla", se dio en el $100 \%$ de las mujeres y en la violencia física grave, la manifestación "la han golpeado con el puño o alguna otra cosa que pudiera herirla" alcanzó la mayoría con un $88,2 \%$.

Otros tipos de violencia (Tabla III). El 100\% de las

Tabla II

CARACTERIZACIÓN DE LA VIOLENCIA EN LAS MUJERES QUE FUERON AGREDIDAS FÍSICAMENTE POR SU PAREJA DURANTE EL EMBARAZO, PUNTA ARENAS $2014(\mathrm{n}=17)$

\begin{tabular}{lcc}
\hline Variable & $\mathrm{n}$ & $\%$ \\
\hline Número de embarazos que fue agredida & 13 & 76,4 \\
En un embarazo & 2 & 11,8 \\
En dos embarazos & 2 & 11,8 \\
En todos los embarazos & & \\
\hline La agresión ocurrió en el último embarazo & 9 & 52,9 \\
Si & 8 & 47,1 \\
No & & \\
\hline Los golpes o patadas fueron en el abdomen, estando embarazadas & 9 & 52,9 \\
Si & 8 & 47,1 \\
No & & \\
\hline La pareja la había golpeado anteriormente & 14 & 82,3 \\
Si & 3 & 17,6 \\
No & & \\
\hline Hijos presentes durante los episodios de violencia & 16 & 94,1 \\
Si & 1 & 5,9 \\
No & 17 & 100,0 \\
\hline Violencia física leve & 17 & 100,0 \\
La han abofeteado o tirado cosas que pudieron herirla & 16 & 94,1 \\
La han empujado, arrinconado o tirado el pelo & 17 & 100,0 \\
Violencia física grave & 15 & 88,2 \\
La han golpeado con el puño u otra cosa que pudiera herirla & 12 & 70,6 \\
La han pateado, arrastrado o dado una golpiza & 12 & 70,6 \\
Han intentado estrangularla a propósito & 0 & 0,0 \\
Han intentado quemarla a propósito & 7 & 41,2 \\
Han amenazado con usar o usado una pistola, cuchillo u otra arma en contra suya & & \\
\hline & & \\
\hline
\end{tabular}


mujeres estudiadas presentó violencia psicológica. "La ha insultado o la ha hecho sentir mal con usted misma", fue la manifestación que se presentó en la totalidad de las mujeres. En relación a la violencia sexual esta se presentó en un $71,4 \%$. La manifestación "quiso que usted tuviera relaciones sexuales cuando usted no lo deseaba y usted lo hizo porque tenía miedo a lo que él le pudiera hacer", alcanzó un 70,6\%. La violencia económica se presentó en un $57,1 \%$

Manifestaciones de violencia física (Tabla IV). Las manifestaciones de violencia física leve "abofeteado o tirado cosas que pudieran herirla" $(p=0,000)$ y violencia física grave "golpeado con su puño u otra cosa que pudiera herirla" $(p=0,006)$, "pateado, arrastrado o dado una golpiza" $(\mathrm{p}=0,004)$, "estrangularla a propósito" $(p=0,010)$, presentaron diferencias altamente significativas en ambos grupos de mujeres, por lo tanto su presencia, se asocia con las mujeres que presentan violencia física durante el embarazo. La presencia de lesiones como producto de la violencia física tiene diferencias significativas en ambos grupos $(p=0,048)$, por lo tanto se relaciona con la presencia de violencia física durante el embarazo por parte de su pareja.

Factores que se relacionan con la presencia de violencia física durante el embarazo (Tabla V). Las variables nivel educacional de la pareja en su categoría media completa o menos $(p=0,047)$, condición laboral de la mujer $(p=0,018)$, indican que existen diferencias significativas en ambos grupos, por lo tanto influyen en la presencia de violencia física durante el embarazo por parte de su pareja. En cambio las variables estrato socioeconómico $(p=0,081)$, apoyo familiar $(p=0,487)$, número de hijos $(p=0,563)$, situación conyugal $(p=0,566)$, deseo del embarazo del último hijo/a en la mujer $(p=0,416)$, deseo del embarazo del último hijo/a en la pareja $(p=0,392)$ y frecuencia de embriaguez en la pareja $(p=0,430)$, indican que no existe diferencia significativa en ambos grupos, por lo tanto no influyen en la presencia de violencia física durante el embarazo en las mujeres en estudio.

La experiencia de violencia física en la mujer antes de los 15 años de edad ( $p=0,301)$, experiencia de abuso sexual en la mujer antes de los 15 años de edad $(p=0,255)$ y antecedentes de golpes en la madre de la mujer, por parte del padre $(p=0,318)$, indican que no existen diferencias significativas en ambos grupos, por lo tanto no influyen en la presencia de violencia física durante el embarazo por parte de su pareja (Tabla VI). En cambio la variable "antecedentes de peleas físicas en la pareja con otros hombres" $(p=0,046)$, indican que existen diferencias significativas en ambos grupos, por lo tanto influye en la presencia de violencia física durante el embarazo por parte de su pareja. DISCUSIÓN

\section{Tabla III \\ DISTRIBUCIÓN DE LAS MUJERES QUE FUERON AGREDIDAS FISICAMENTE POR SU PAREJA DURANTE EL EMBARAZO, SEGÚN MANIFESTACIONES DE VIOLENCIA PSICOLÓGICA Y SEXUAL, PUNTA ARENAS 2014}

\begin{tabular}{lcc} 
& Con violencia física durante el embarazo \\
\hline Violencia psicológica & $\mathrm{n}$ & $\%$ \\
La ha insultado o la ha hecho sentir mal con Ud. misma & 17 & 100 \\
La ha menospreciado o humillado frente a otras personas & 17 & 100 \\
Él ha hecho cosas a propósito para asustarla o intimidarla & 15 & 88,2 \\
La han amenazado con herirla o a alguien que usted le importa & 15 & 88,2 \\
\hline & 12 & 70,6 \\
\hline $\begin{array}{l}\text { Violencia sexual } \\
\text { La han forzado físicamente a tener relaciones sexuales cuando usted no }\end{array}$ & 12 & 70,6 \\
lo deseaba & 10 & 54,4 \\
$\begin{array}{l}\text { Quiso que usted tuviera relaciones sexuales cuando usted no lo deseaba } \\
\text { y usted lo hizo porque tenía miedo a lo que él le pudiera hacer } \\
\text { La forzó alguna vez a realizar algún acto sexual que usted encontró }\end{array}$ & 12 & 70,6 \\
humillante & 5 & 29,4 \\
\hline
\end{tabular}




\section{Tabla IV \\ MANIFESTACIONES DE VIOLENCIA FÍSICA LEVE Y GRAVE QUE SE ASOCIAN CON LA PRESENCIA DE VIOLENCIA FÍSICA DURANTE EL EMBARAZO}

\begin{tabular}{|c|c|c|c|c|c|c|}
\hline \multirow[b]{2}{*}{ Manifestaciones } & \multicolumn{2}{|c|}{$\begin{array}{l}\text { Sin violencia } \\
\text { física durante el } \\
\text { embarazo }\end{array}$} & \multicolumn{2}{|c|}{$\begin{array}{l}\text { Con violencia } \\
\text { física durante el } \\
\text { embarazo }\end{array}$} & \multirow[b]{2}{*}{ Estadígrafo } & \multirow[b]{2}{*}{ Valor $\mathrm{p}$} \\
\hline & $\mathrm{n}$ & $\%$ & $\mathrm{n}$ & $\%$ & & \\
\hline \multicolumn{7}{|l|}{ Violencia física leve } \\
\hline $\begin{array}{l}\text { Abofeteado o tirado cosas que pudieran } \\
\text { herirla }\end{array}$ & & & & & $11,343 a$ & $0,000^{\star \star \star}$ \\
\hline $\mathrm{Si}$ & 23 & 54,8 & 17 & 100,0 & & \\
\hline No & 19 & 45,2 & 0 & 0,0 & & \\
\hline \multicolumn{7}{|l|}{ Violencia física grave } \\
\hline $\begin{array}{l}\text { Golpeado con su puño o con alguna otra } \\
\text { cosa que pudiera herirla }\end{array}$ & & & & & $7,438 a$ & $0,006^{\star \star}$ \\
\hline $\mathrm{Si}$ & 21 & 50,0 & 15 & 88,2 & & \\
\hline No & 21 & 50,0 & 2 & 11,8 & & \\
\hline Pateado, arrastrado o dado una golpiza & & & & & $8,854 a$ & $0,004^{* *}$ \\
\hline $\mathrm{Si}$ & 12 & 28,6 & 12 & 70,6 & & \\
\hline No & 30 & 71,4 & 5 & 29,4 & & \\
\hline Estrangularla a propósito & & & & & $6,814 a$ & $0.010^{*}$ \\
\hline $\mathrm{Si}$ & 14 & 33,3 & 12 & 70,6 & & \\
\hline No & 28 & 66,7 & 5 & 29,4 & & \\
\hline $\begin{array}{l}\text { Lesiones como producto de la violencia } \\
\text { física }\end{array}$ & & & & & $3,779 a$ & $0,048^{*}$ \\
\hline $\mathrm{Si}$ & 29 & 70,7 & 16 & 94,1 & & \\
\hline No & 12 & 29,3 & 1 & 5,9 & & \\
\hline
\end{tabular}

$a=$ Chi-cuadrado. Significancia que se expresa en ${ }^{*} p<0,05{ }^{* *} p<0,01{ }^{* * *} p<0,001$

La agresión física durante el embarazo reviste una connotación de gravedad, ya que no solo provoca consecuencias en la salud mental y física de la mujer, sino que también en el hijo que está en gestación. Del grupo de mujeres estudiadas, el $28,8 \%$, declaró haber sido agredida físicamente mientras estaban embarazadas, esta cifra es mayor a los hallazgos encontrados en estudios internacionales $(11,12,13-$ 15) y en estudios nacionales (16-19) y menor al estudio de Costa Rica (10) y México (14). El que la prevalencia encontrada haya sido mayor que la mayoría de los estudios, puede deberse a que las mujeres en estudio están adscritas a un programa de violencia lo que puede favorecer las respuestas y reconocimiento de este tipo de violencia.

El perfil de las mujeres que presentaron violencia física durante el embarazo, el mayor porcentaje se encontraba en el grupo de edad entre 15 a 29 años, coincidente con estudios internacionales (10,11-14) y no similar (13-15), y al estudio nacional de la región de Valdivia (16) y Temuco (17), donde las edades eran mayores a las encontradas. Estas similitudes y diferencias pueden deberse al momento en que se realizan los estudios. La mayoría había completado la enseñanza media, es decir, 12 años de instrucción, similar a estudios internacionales (12-15) y de la región de Valdivia (16) y a diferencia de estudios en Costa Rica $(10)$, Brasil $(11,28)$, donde la situación de violencia se da en mujeres con bajo nivel de escolaridad, es decir, menos de 8 años de instrucción. Esta situación se puede deber a que las mujeres estudiadas tendrían más acceso a información y mayores recursos personales que las han llevado a solicitar ayuda, ya que todas están adscritas a un programa de violencia, sumado a que se encuentran trabajando fuera del hogar, similar al estudio de México (13) y de la región de Valdivia (16) y diferente a los estudios de Costa Rica (10) y México (14).

El trabajar fuera del hogar puede entregar a la 


\section{Tabla V \\ FACTORES QUE SE RELACIONAN CON LA PRESENCIA DE VIOLENCIA FÍSICA DURANTE EL EMBARAZO}

\begin{tabular}{|c|c|c|c|c|c|c|}
\hline \multirow[b]{2}{*}{ Variable } & \multicolumn{2}{|c|}{$\begin{array}{c}\text { Sin violencia } \\
\text { física durante el } \\
\text { embarazo }\end{array}$} & \multicolumn{2}{|c|}{$\begin{array}{l}\text { Con violencia } \\
\text { física durante el } \\
\text { embarazo }\end{array}$} & \multirow[b]{2}{*}{ Estadígrafo } & \multirow[b]{2}{*}{ Valor $p$} \\
\hline & $\mathrm{n}$ & $\%$ & $\mathrm{n}$ & $\%$ & & \\
\hline Estrato socioeconómico & & & & & $2,849 a$ & 0,081 \\
\hline Bajo, Muy-bajo & 25 & 59,5 & 6 & 35,3 & & \\
\hline Apoyo familiar & & & & & $0,104 a$ & 0,487 \\
\hline $\mathrm{Si}$ & 29 & 69,0 & 11 & 64,7 & & \\
\hline No & 13 & 31,0 & 6 & 35,3 & & \\
\hline Número de hijos & & & & & $0,067 b$ & 0,563 \\
\hline Un hijo & 2 & 11,8 & 4 & 9,5 & & \\
\hline Dos hijos o más & 15 & 88,2 & 38 & 90,5 & & \\
\hline Situación conyugal & & & & & $1,416 b$ & 0,566 \\
\hline Conviviente & 16 & 38,1 & 9 & 52,9 & & \\
\hline Casada & 25 & 59,5 & 8 & 47,1 & & \\
\hline Separada/pololo & 1 & 2,4 & 0 & 0,0 & & \\
\hline Nivel educacional en la pareja & & & & & $4,417 a$ & $0,047^{\star}$ \\
\hline Educación media o menos & 32 & 78,0 & 17 & 100 & & \\
\hline $\begin{array}{l}\text { Educación superior incompleta } \\
\text { o completa }\end{array}$ & 9 & 22,0 & 0 & 0,0 & & \\
\hline $\begin{array}{l}\text { Deseo del embarazo del último hijo/a } \\
\text { en la mujer }\end{array}$ & & & & & $2,871 b$ & 0,416 \\
\hline Quedar embarazada en ese momento & 20 & 48,8 & 6 & 35,3 & & \\
\hline Hubiera querido esperar & 6 & 14,6 & 1 & 5,9 & & \\
\hline No quería hijos & 14 & 34,2 & 9 & 52,9 & & \\
\hline Le daba lo mismo & 1 & 2,4 & 1 & 5,9 & & \\
\hline $\begin{array}{l}\text { Deseo del embarazo del último hijo/a en } \\
\text { la pareja }\end{array}$ & & & & & $3,049 b$ & 0,392 \\
\hline Quedar embarazada en ese momento & 18 & 47,4 & 5 & 33,3 & & \\
\hline Hubiera querido esperar & 6 & 15,8 & 1 & 6,7 & & \\
\hline No quería hijos & 10 & 26,3 & 5 & 33,3 & & \\
\hline Le daba lo mismo & 4 & 10,5 & 4 & 26,7 & & \\
\hline Frecuencia de embriaguez en la pareja & & & & & $1850 a$ & 0,430 \\
\hline Raramente & 16 & 38,1 & 4 & 23,5 & & \\
\hline De vez en cuando & 7 & 16,7 & 2 & 11,8 & & \\
\hline Frecuentemente & 19 & 45,2 & 11 & 64,7 & & \\
\hline Condición laboral de la mujer & & & & & $9,101 b$ & $0,018^{*}$ \\
\hline Trabajo remunerado fuera del hogar & 21 & 50,0 & 10 & 58,8 & & \\
\hline Trabajo remunerado en el hogar & 2 & 4,8 & 5 & 29,4 & & \\
\hline Trabajo remunerado esporádico & 4 & 9,5 & 0 & 0,0 & & \\
\hline Sin actividad laboral/estudia & 15 & 35,7 & 2 & 11,8 & & \\
\hline
\end{tabular}

$a=$ Chi-cuadrado. $b=$ Test exacto de Fisher. Significancia que se expresa en ${ }^{*} p<0,05^{* *} p<0,01{ }^{* * *} p<0,001$ 


\section{Tabla VI}

\section{FACTORES DE EXPERIENCIAS PREVIAS DE VIOLENCIA QUE SE RELACIONAN CON LA PRESENCIA DE VIOLENCIA FÍSICA DURANTE EL EMBARAZO}

\begin{tabular}{|c|c|c|c|c|c|c|}
\hline \multirow[b]{2}{*}{ Variable } & \multicolumn{2}{|c|}{$\begin{array}{c}\text { Sin violencia } \\
\text { física durante el } \\
\text { embarazo }\end{array}$} & \multicolumn{2}{|c|}{$\begin{array}{l}\text { Con violencia } \\
\text { física durante el } \\
\text { embarazo }\end{array}$} & \multirow[b]{2}{*}{ Estadígrafo } & \multirow[b]{2}{*}{ Valor $\mathrm{p}$} \\
\hline & $\mathrm{n}$ & $\%$ & $\mathrm{n}$ & $\%$ & & \\
\hline $\begin{array}{l}\text { Peleas físicas de la pareja con otros } \\
\text { hombres }\end{array}$ & & & & & $3,952 a$ & $0,046^{\star}$ \\
\hline $\mathrm{Si}$ & 13 & 31,0 & 10 & 58,8 & & \\
\hline No & 29 & 69,0 & 7 & 41,2 & & \\
\hline $\begin{array}{l}\text { Experiencia de violencia física en la mujer } \\
\text { antes de los } 15 \text { años }\end{array}$ & & & & & $0,655 a$ & 0,301 \\
\hline $\mathrm{Si}$ & 15 & 35,7 & 8 & 47,1 & & \\
\hline No & 27 & 64,3 & 9 & 52,9 & & \\
\hline $\begin{array}{l}\text { Experiencia de abuso sexual en la mujer } \\
\text { antes de los } 15 \text { años }\end{array}$ & & & & & $0,920 a$ & 0,255 \\
\hline $\mathrm{Si}$ & 18 & 42,9 & 5 & 29,4 & & \\
\hline No & 24 & 57,1 & 12 & 70,6 & & \\
\hline $\begin{array}{l}\text { Antecedentes de golpes en la madre de } \\
\text { la mujer, por parte del padre }\end{array}$ & & & & & $0,948 a$ & 0,318 \\
\hline $\mathrm{Si}$ & 19 & 70,4 & 7 & 87,5 & & \\
\hline No & 8 & 29,6 & 1 & 12,5 & & \\
\hline
\end{tabular}

$a=$ Chi-cuadrado. Significancia que se expresa en ${ }^{*} p<0,05^{\star \star} p<0,01{ }^{* \star \star} p<0,001$

mujer, autonomía económica y mayores recursos personales para solicitar en este caso ayuda, pero también puede ser detonante de situaciones de violencia por parte de la pareja, al ver que está perdiendo el control de la mujer, al tener ella mayor independencia, tanto en lo económico como en la toma de decisiones. Esto probablemente también se relaciona con el mayor porcentaje de mujeres que presenta un nivel socioeconómico medio, medio-bajo, similar al estudio de Perú (15) y de Valdivia (16).

El mayor porcentaje de las mujeres se encontraba conviviendo, similar a la región de Valdivia (16) y diferente al estudio mexicano (14). Esta situación probablemente se presentó, ya que las mujeres son mayoritariamente jóvenes y estas más bien optan por una relación de convivencia antes de establecer relaciones más formales, como el matrimonio, pudiendo decidir cesar la relación sin mayores trámites legales.

La mayoría de las mujeres tiene dos o más hijos, similar al estudio de Brasil (11). El hecho de tener dos o más hijos, puede aumentar la dependencia económica de la mujer y los conflictos entre la pareja, y traer dificultades en cuanto a la posibilidad de salir de la situación en donde puede además existir poco apoyo social para el cuidado de los niños, soportando de esta manera la situación de violencia durante el embarazo (29).

El $94,1 \%$ de los hijos han estado presentes en los episodios de violencia, cifra mucho mayor que estudios de las regiones de Coquimbo (24), Metropolitana y Araucanía (30) y Aysén (31), donde los porcentajes oscilaban entre el $61,5 \%$ y $67,5 \%$, y menor que la región de los Lagos (18), donde alcanzo un $95 \%$ de presencia de los hijos en las situaciones de violencia. Estos hallazgos son alarmantes, ya que estudios (32), han demostrado que disminuyen su sensibilidad interpersonal, es decir pierden la capacidad de comprender las situaciones sociales y los pensamientos de las personas que intervienen en dichas situaciones en comparación con niños que no han estado expuestos a situaciones de violencia materna, además se asocia con conductas de alto riesgo, como la práctica del sexo no seguro en etapas 
posteriores de la vida.

En relación a las características de la violencia física durante el embarazo, se puede destacar que esta agresión había sido perpetrada en un alto porcentaje por el padre del hijo que estaba esperando, siendo similar a estudios internacionales (10-13) y nacional de la región de los Lagos (18). El 82\% de estas mujeres había sido golpeada por su pareja antes de estar embarazada, esto concuerda con estudios mencionados anteriormente y principalmente con el estudio de México (13), donde la probabilidad de presentar violencia durante el embarazo es 4,5 veces más en mujeres con experiencias previas de violencia en la relación de pareja. El que la violencia en contra de la mujer también se produzca durante el embarazo, puede darnos cuenta que el patrón de conducta violenta en la pareja es permanente y que no cesa incluso en una situación de riesgo en la mujer, como en este caso es el embarazo. En más de la mitad de las mujeres los golpes o patadas fueron en el abdomen, lo que aumenta el riesgo de complicaciones en la mujer y el hijo. Sin duda este tipo de violencia adquiere una connotación especial por la gravedad a la que está expuesta la mujer, la que se asocia con abortos, muerte fetal, parto prematuro, muerte y lesiones fetales del recién nacido (12).

La totalidad de las mujeres estudiadas, que presentaban violencia física durante el embarazo, presentaron en algún momento de la relación, violencia psicológica, física leve y grave, esto no es comparable con estudios internacionales 0 nacionales, donde no se especifica el momento de ocurrida la situación violenta y tampoco las manifestaciones presentes. Sin embargo el tipo de violencia que adquiere siempre el mayor porcentaje, es la de tipo psicológico, seguido por la física y por último la sexual. Si es preocupante que al comparar estos resultados con las mujeres del estudio que no presentaron violencia física durante el embarazo, los porcentajes son alarmantes, principalmente cuando la presencia de violencia física grave, está presente en el $100 \%$ de las mujeres. Sumado a esto, podemos decir que 4 de las 5 manifestaciones de violencia física grave, se relacionan significativamente con la presencia de violencia física durante el embarazo, al igual que la presencia de lesiones producto del acto violento. Esto adquiere gran relevancia y preocupación, si tenemos en consideración que el femicidio es la forma más extrema de violencia contra las mujeres. En el año 2013, en Chile, se cometieron 40 femicidios y al mes de junio de 2014 ya van 20 y donde probablemente, en dos de ellos, la mujer se encontraba embarazada (33). El penúltimo femicidio, se produjo en la ciudad de Punta Arenas, lugar del estudio, la víctima tenía 24 años y con una denuncia anterior por violencia. Estas situaciones extremas en este tipo de violencia, nos confirma que es responsabilidad de todas las personas que trabajamos en salud de detectar en forma precoz la violencia en contra de la mujer por parte de su pareja.

La violencia sexual también estuvo presente en las mujeres que presentaron violencia física durante el embarazo, alcanzando el $70,6 \%$, cifra mucho mayor al estudio de Costa Rica (10) y México (13). El hecho de que las mujeres pertenezcan a un programa de violencia, facilita el reconocimiento de manifestaciones de violencia psicológica, que en muchas ocasiones se consideran normales como parte de la relación de pareja, a esta situación, puede deberse el alto porcentaje en este tipo de violencia en las mujeres en estudio.

Los factores que se relacionaron con la presencia de violencia física durante el embarazo en este grupo de mujeres fueron: educación media o menos de la pareja, trabajo remunerado de la mujer en el hogar, participación de la pareja en peleas físicas con otro hombre y presencia de manifestaciones de violencia física leve y grave y lesiones producto de la violencia. Estos resultados no son comparables con estudios internacionales, ya que estos indagaron la relación de factores en el momento que las mujeres estaban embarazadas y viviendo la situación de violencia, a diferencia del momento en que se obtuvieron los datos en este estudio. Si es comparable con el estudio de Temuco (17), siendo diferentes los factores que resultaron relacionados, estos fueron existencia de violencia entre los padres, bajo nivel de escolaridad, carencia de empleo remunerado, consumo de alcohol y falta de redes de apoyo familiar. Esta situación se puede deber a que la población estudiada fue muy diferente, ya que en el estudio de Temuco fue una comunidad de nivel socioeconómico medio-bajo, situación que puede condicionar otros factores como nivel educacional, condición laboral, acceso a mayor información y mayores recursos personales, entre otros.

\section{CONCLUSIONES}

Este estudio proporciona los primeros resultados en mujeres que presentaron violencia física durante el embarazo y que se encuentran adscritas a los programas de violencia en la ciudad de Punta Arenas, en relación a perfil, prevalencia y tipos de violencia. Se encontró una prevalencia 
mayor a estudios internacionales y nacionales. Los factores que se relacionaron a la presencia de violencia física durante el embarazo fueron: educación media o menos de la pareja, trabajo remunerado de la mujer en el hogar, participación de la pareja en peleas físicas con otro hombre, presencia de manifestaciones de violencia física leve y grave y lesiones producto de la violencia. Los hallazgos obtenidos nos permiten visibilizar el fenómeno de la violencia física durante el embarazo en la comuna de Punta Arenas y la importancia de identificar precozmente a las mujeres embarazadas con mayor riesgo, con la finalidad de ofrecer una intervención oportuna, minimizando los efectos negativos en la mujer y la repercusión que esto podría tener en sus hijos.

\section{REFERENCIAS}

1. Organización de Naciones Unidas. Declaración sobre la eliminación de la violencia contra las mujeres (Res. AG/48/104). Nueva York: Naciones Unidas.

2. Organización de las Naciones Unidas. Convención sobre la Eliminación de todas las formas de Discriminación contra la Mujer. Adoptada y abierta a la firma y ratificación, o adhesión, por la Asamblea General en su resolución 34/180, de 18 de diciembre de 1979. Entrada en vigor: 3 de septiembre de 1981, de conformidad con el artículo 27. Disponible en: http://www.unhchr.ch/tbs/doc.nsf/(Symbol)/3c3559ce 607fb470c1256a3300549df2?Opendocument

3. Ministerio de Salud de Chile. Política de Equidad de Género en Salud. Santiago 16 de febrero de 2010 , Exento №12.

4. Ministerio de Salud de Chile. Política de Salud en Violencia de Género. Santiago 16 de mayo de 2008, Exenta, № 276.

5. Collado Peña SP, Villanueva Egan LA. Relación entre la violencia doméstica durante el embarazo y el riesgo de bajo peso en el recién nacido. Ginecol Obstet Mex 2007;75:259-67.

6. Watts C, Keogh E, Ndlovu M, Kwaramba R. Withholding of sex and forced sex: dimensions of violence against Zimbabwean women. Reprod Health Matters 1998;6:57-65.

7. Gazmararian JA, Lazorick S, Spitz AM, Ballard TJ, Saltzman LE, Marks JS. Prevalence of violence against pregnant women. JAMA 1996;275:1915-20.

8. Moraes CL, Reichenheim ME. La violencia doméstica durante el embarazo, en Río de Janeiro, Brasil. Int J Obstet Gynaecol 2002;79:269-77.

9. Audi CA, Segall-Corrêa AM, Santiago SM, Andrade $M$ da $G$, Pérez-Escamila R. La violencia contra las mujeres embarazadas: prevalencia y factores asociados. Rev Saúde Pública. 2008;42:877-85.

10. Nuñez-Rivas H, Monge-Rojas R, Gríos-Dávila $C$, Elizondo-Ureña AM, Rojas-Chavarra A. La violencia física, psicológica y sexual durante el embarazo: riesgo reproductivo predictor de bajo peso al nacer en
Costa Rica. Rev Panam Salud Pública 2003;14:7583.

11. Collado S, Villanueva LA. Violencia familiar: una aproximación desde la ginecología y obstetricia. Rev Gineco IObstet Mex 2005;73:250-60.

12. Castro R, Ruiz A. Prevalencia y severidad de la violencia contra las mujeres embarazadas. Rev Saúde Pública 2004;38:62-70.

13. Cuevas $S$, Blanco $C$, Juárez $C$, Palma $O$, ValdezSantiago R. Violencia y embarazo en usuarias del sector salud en estados de alta marginación en México. Rev Salud Pública México 2006;48:239-49.

14. Doubova S, Pámanes-Gonzalez V, Billings D, Torres-Arreola L. Violencia de pareja en mujeres embarazadas en la ciudad de México. Rev Saúde Pública 2007;41: 52-90.

15. Saravia JC, Mejía M, Becerra S, Palomino A. Violencia física contra la mujer durante el embarazo: prevalencia y factores asociados. Rev Peru Epidemiol 2012;16:84-90.

16. Arcos E, Molina I, Repossi A, Uarac M, Ritter P, Arias L. Violencia doméstica y sexualidad. Rev Méd Chile 1999;127:1329-38.

17. Vizcarra L María Beatriz, Cortés M Julia, Bustos M Luis, Alarcón E Marina, Muñoz N Sergio. Violencia conyugal en la ciudad de Temuco: Un estudio de prevalencia y factores asociados. Rev Méd Chile 2001;129:1405-12.

18. Servicio Nacional de la Mujer (SERNAM). Documento de trabajo №106 Detección y Análisis de la Prevalencia de la Violencia Intrafamiliar en la Región de los Lagos. Santiago: Depto de Estudios y Capacitación; 2009.

19. Bohle X. Magnitud y características de la violencia doméstica en las mujeres embarazadas, atendidas en el servicio de obstetricia y ginecología del Hospital Clínico Regional de Valdivia [Tesis de grado de Licenciado en Obstetricia y Puericultura]. Valdivia. Chile. Universidad Austral de Chile; 2008.

20. Krug EG, Dahlberg LL, Mercy JA, Zwi AB, Lozano R. World Report on Violence and Health. Ginebra. Organización Mundial de la Salud (OMS). Publicación Científica y Técnica $N^{\circ} 588.2002$.

21. Corporación Municipal de Punta Arenas. Dpto de Estadística. Disponible en: http://www.cormupa.cl/ cormupa/consultorios.php. Acceso: 2 de diciembre de 2013.

22. Ruiz A. Coordinadora de la Unidad Regional de Prevención de la Violencia Intrafamiliar contra la mujer. SERNAM, región de Magallanes y Antártica Chilena.

23. Organización Mundial de la Salud. Estudio Multipaís de la OMS sobre salud de la mujer y experiencias de la vida. Cuestionario Final, versión 10. Ginebra: Departamento de Género, Mujer y Salud Familiar y Comunitaria OMS. 2005.

24. Ruz M, Larraín S, Madrid A, Fernández M. Detección y análisis de la prevalencia de la violencia intrafamiliar en la Región de Coquimbo. Coquimbo: Dirección de Estudios Sociológicos de la Pontificia Universidad Católica y SERNAM; 2004.

25. Emanuel E, Wendler D, Killen J, Grady C. What makes clinical research in developing countries ethical? The 
bench mark of ethical research. Perspective $\mathrm{J}$ Infect Dis 2004;189:930-7.

26. World Health Organization. Putting Women's Safety First: Ethical and Safety Recommendations for Research on Domestic Violence Against Women. Geneva: Global Programme on Evidence for Health Policy, World Health Organization; 1999.

27. Arratia A. Aspectos éticos vulnerados en situaciones de violencia: su importancia en la formación de enfermería. Invest Educ Enferm 2005;23:104-16.

28. Fernández E, Granado S, Lázaro M, Wernersbach L. Los factores asociados con la agresión física en las mujeres embarazadas y los resultados adversos para el recién nacido. J Pediatr 2013;1:83-90.

29. Meloni E, Castro G, Manoel dos Santos A. Factores asociados a la violencia física por pareja íntima en usuarias de los servicios de salud. Rev Saude Pública 2011;45:730-7.

30. Servicio Nacional de la Mujer (SERNAM).
Documento de trabajo №121. Detección y Análisis de la Prevalencia de la Violencialntrafamiliar en la RegiónMetropolitana y La Araucanía. Depto. de Estudios y Capacitación. Santiago. 2009.

31. Dirección de Estudios Sociológicos de la Pontificia Universidad Católica de Chile (DESUC). Detección y análisis de la Violencia Intrafamiliar en la región de Aysén. Informe Final. Aysén: SERNAM; 2006.

32. García-Moreno C. Violencia contra la mujer: género y equidad en la salud. Harvard Center for Population and Development Studies. Publicación Ocasional №6. Organización Panamericana de la Salud. 2000.

33. Servicio Nacional de la Mujer. SERNAM. Femicidios en Chile. Disponible: http://www.sernam.cl/portal/ index.php/femicidios 2013-2014. 